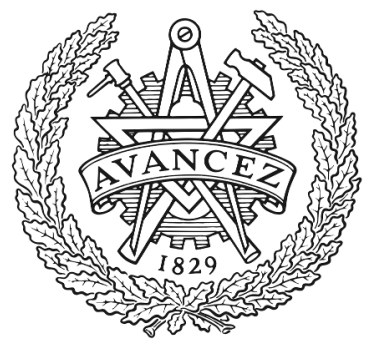

CHALMERS

UNIVERSITY OF TECHNOLOGY

\title{
Joint Phase Tracking for Multicore Transmission with Correlated Phase Noise
}

Downloaded from: https://research.chalmers.se, 2023-04-26 15:14 UTC

Citation for the original published paper (version of record):

Alfredsson, A., Agrell, E., Wymeersch, H. et al (2018). Joint Phase Tracking for Multicore

Transmission with Correlated Phase Noise. [Source Title missing]: 16-17.

http://dx.doi.org/10.1109/PHOSST.2018.8456661

N.B. When citing this work, cite the original published paper. 


\title{
Joint Phase Tracking for Multicore Transmission with Correlated Phase Noise
}

\author{
Arni F. Alfredsson ${ }^{\dagger}$, Erik Agrell ${ }^{\dagger}$, Henk Wymeersch ${ }^{\dagger}$, \\ Benjamin J. Puttnam ${ }^{\ddagger}$, and Ruben S. Luís \\ ${ }^{\dagger}$ Department of Electrical Engineering, Chalmers University of Technology, Gothenburg, SE-412 96, Sweden \\ ${ }^{\ddagger}$ Photonic Network System Laboratory, NICT, 4-2-1 Nukui-Kitamachi, Koganei, Tokyo 184-8759, Japan \\ arnia@chalmers.se
}

\begin{abstract}
Space-division multiplexed transmission over multicore fibers offers potential for joint-core processing to compensate for correlated phase noise. We review methods that take advantage of the phase-noise correlation across cores and assess their benefits in terms of transmission reach and pilot-rate requirements.
\end{abstract}

OCIS codes: 060.1660 Coherent communications, 060.2330 Fiber optics communications.

\section{Introduction}

In order to cope with the ever-increasing throughput demands on fiber-optical transmission systems, space-division multiplexing (SDM) using multimode or multicore fibers (MCFs) is recognized as a viable option to extend the capacity of these systems in a cost-effective way [1]. One of the appealing aspects of SDM is the sharing of hardware resources at the transmitters and receivers, including transmitter lasers and local oscillators. In these cases, the impact of the phase noise on the detected signals becomes correlated [2], which suggests the use of joint digital signal processing (DSP) to improve signal quality. This is particularly relevant when high-order modulation formats are used, as transmission generally becomes more sensitive to phase noise as the modulation order grows.

\section{Phase Tracking Strategies}

In case of transmission over MCFs with shared lasers at the transmitter and receiver sides, multiple phase-tracking strategies can be used, some of which exploit the correlation in the phase noise, which can lead to reduced complexity or increased phase tracking performance. We review and compare three strategies that can be carried out in DSP using pilot-aided [3] or blind [4] algorithms. In this work, we focus on pilot-aided phase tracking.

Separate channel processing: The conventional approach involves performing phase tracking separately on each channel [4], which is necessary if the phase noise is independent between channels. However, in the case of correlated phase noise, computational complexity and phase tracking performance remain unchanged.

Master-slave processing: In this strategy, a selected channel is designated as a master channel. Phase tracking is performed on this channel and the phase-noise estimates are used to correct for the phase noise in all remaining channels, i.e., the slave channels [2]. This strategy allows for a computational complexity reduction in DSP compared with separate channel processing, as phase tracking is only performed on one channel, regardless of the number of channels. However, perfect phase-noise correlation is implicitly assumed in this strategy and any phase drift between channels will quickly degrade its performance.

Joint channel processing: This method uses observations on all channels to jointly estimate their phase noise. In other words, the observations of phase noise on each channel influence the phase tracking of other channels. The phase-noise correlation must be known or estimated, as it governs how much the phase-tracking influence between channels should be. In the case of independent phase noise in each channel, this strategy reduces to separate channel processing. In contrast, with perfectly correlated phase noise, this strategy essentially carries out estimate averaging across the channels. Hence, the phase-tracking performance can be increased for a fixed pilot rate, or reversely, the pilot rate can be reduced without sacrificing phase-tracking performance. However, these improvements may come at the cost of increased computational complexity in DSP compared to separate channel processing.

\section{Experimental Results}

We experimentally validated and compared the different phase-tracking strategies in terms of bit error rate (BER) performance versus transmission distance. The experimental setup consisted of 3 synchronized recirculating loops running through 3 cores of a $53.7 \mathrm{~km}$ 7-core weakly-coupled single-mode MCF. The transmitted signals were dualpolarization quadrature phase-shift keying (DP-QPSK), DP 16-ary quadrature amplitude modulation (DP-16QAM), 


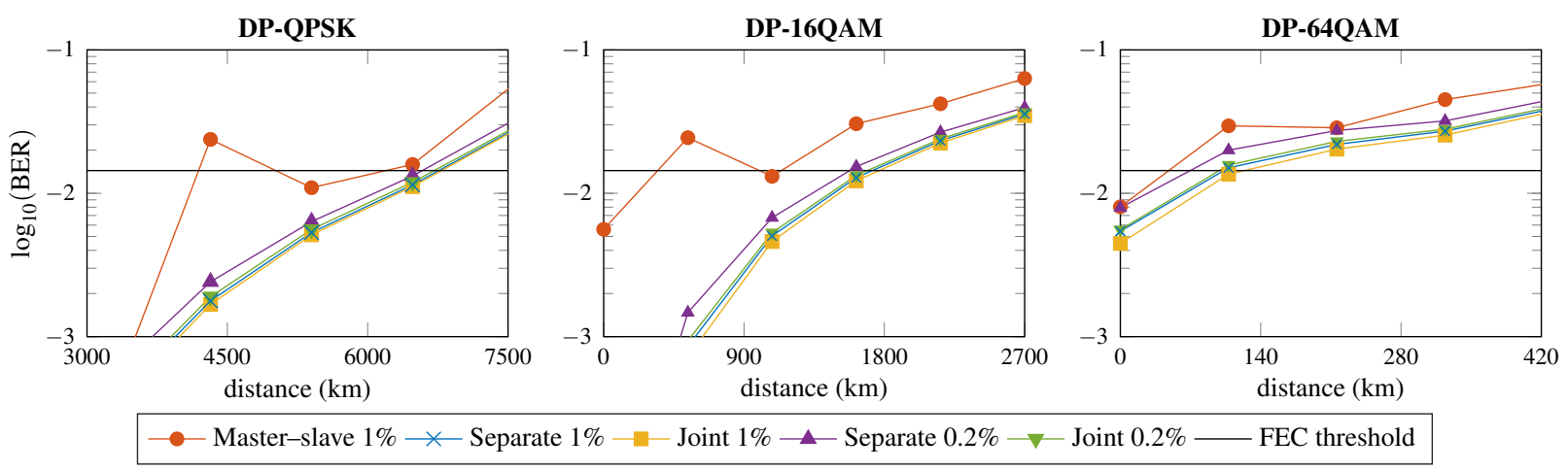

Fig. 1. BER performance versus transmission distance for the different phase-tracking strategies, evaluated at $1 \%$ and $0.2 \%$ pilot rates for transmission of DP-QPSK, DP-16QAM, and DP-64QAM.

and DP-64QAM at 20 GBaud. At the transmitter and receiver sides, an external cavity laser with $100 \mathrm{kHz}$ linewidth operating at $1550 \mathrm{~nm}$ was shared for all cores. For a more detailed description of the setup, refer to [5]. The detected signal was stored at $80 \mathrm{GS} / \mathrm{s}$ for offline DSP using MATLAB and C. All stages in the DSP chain except for phase tracking were applied separately on each core. The first stages in the chain consisted of resampling to 2 samples per symbol, dispersion compensation, orthonormalization, timing recovery, and blind $2 \times 2$ equalization using the constant modulus algorithm to allow for frame synchronization. This was followed by a training stage using approximately $10^{5}$ symbols for least mean square $2 \times 2$ equalization and estimation of the complex additive noise variance. After the training stage, blind equalization was performed using a radially-directed equalizer followed by blind frequency-offset estimation, down-conversion, matched filtering, and down-sampling to 1 sample per symbol. The final stage consisted of pilot-aided phase tracking using the aforementioned strategies and a second orthonormalization step to correct for transmitter I/Q imbalance. All phase-tracking strategies were implemented using a previously developed pilot-aided joint phase tracking algorithm [6]. In case of separate phase tracking and the master-slave strategy, the pilot symbols were spread uniformly throughout the transmitted symbol blocks. For joint phase tracking, the pilots were arranged in a wrapped-diagonal fashion, as detailed in [7]. Finally, each BER estimate was computed by counting bit errors out of at least $10^{7}$ bits.

The phase-tracking strategies are evaluated with $1 \%$ and $0.2 \%$ pilot rates for all modulation formats; the results are shown in Fig. 1. Overall, the master-slave strategy sees large penalties due to residual phase drifts that decorrelate the phase noise between the cores. As only one channel is used to obtain phase-noise estimates, other channels are not compensated properly, which leads to a large increase in the average BER. Moreover, through joint phase tracking, the pilot rate can in this case be reduced by a factor of 5 with marginal BER penalty. With a higher number of cores, the pilot rate can be reduced even further while maintaining BER performance. Finally, we assume the use of a harddecision FEC at $20 \%$ overhead, giving a pre-FEC BER threshold of $1.44 \cdot 10^{-2}$ [8]. For $1 \%$ pilot rate, the use of joint phase tracking extends transmission reach by $31 \mathrm{~km}(0.5 \%), 46 \mathrm{~km}(2.7 \%)$, and $20 \mathrm{~km}(19.9 \%)$ for DP-QPSK, DP16QAM, and DP-64QAM transmission, respectively, compared to separate channel processing. For $0.2 \%$ pilot rate, transmission reach is extended by $135 \mathrm{~km}(2.1 \%), 113 \mathrm{~km}(7.2 \%)$, and $40 \mathrm{~km}(75.6 \%)$, respectively.

\section{References}

1. E. Agrell et al., "Roadmap of optical communications," Journal of Optics 18, 063,002 (2016).

2. M. D. Feuer et al., "Joint digital signal processing receivers for spatial superchannels," IEEE Photonics Technol. Lett. 24, 1957-1960 (2012).

3. M. Magarini, L. Barletta, A. Spalvieri, F. Vacondio, T. Pfau, M. Pepe, M. Bertolini, and G. Gavioli, "Pilot-symbols-aided carrier-phase recovery for 100-G PM-QPSK digital coherent receivers," IEEE Photonics Technol. Lett. 24, 739-741 (2012).

4. T. Pfau et al., "Hardware-efficient coherent digital receiver concept with feedforward carrier recovery for $M$-QAM constellations," J. Lightw. Technol. 27, 989-999 (2009).

5. R. S. Luís, B. J. Puttnam, G. Rademacher, Y. Awaji, and N. Wada, "On the use of high-order MIMO for long-distance homogeneous single-mode multicore fiber transmission," in "Proc. Opt. Fiber Commun. Conf.", (2017).

6. A. F. Alfredsson et al., "Iterative decoding and phase-noise compensation for multichannel optical transmission," arXiv e-prints (2018).

7. A. F. Alfredsson et al., "Pilot distributions for phase tracking in space-divison multiplexed systems," in "European Conf. on Optical Communication," (2017).

8. L. M. Zhang and F. R. Kschischang, "Staircase codes with 6\% to 33\% overhead," J. Lightw. Technol. 32, 1999-2002 (2014). 